\title{
BOUNDED GROUPS OF TRANSFORMATIONS
}

\author{
R. C. T. SMITH
}

This note is intended as a comment on a result of Bochner and Martin's and their notation will be used. ${ }^{1}$

Formal power series mapping an $n$-dimensional complex space into itself are considered. A typical inner transformation $T$ mapping $x=\left(x_{1}, \cdots, x_{k}\right)$ into $y=\left(y_{1}, \cdots, y_{k}\right)$ is given by

$$
\begin{aligned}
& y_{j}=C_{1}^{j} x_{1}+\cdots+C_{k}^{j} x_{k}+\sum_{n_{1}+\cdots+n_{k}>1} C_{n_{1} \cdots n_{k}}^{j} x_{1}^{n_{1}} \cdots x_{k}^{n_{k}}, \\
& j=1, \cdots, k \text {. }
\end{aligned}
$$

The linear part $L(T)$ of $T$ is the transformation

$$
y_{j}=C_{1}^{j} x_{1}+\cdots+C_{k}^{i} x_{k} .
$$

Transformations with determinants $\left|C_{i}^{j}\right|$ nonzero form a group where the group operation is defined in the obvious way in terms of successive substitutions.

A group of transformations $T(\alpha)$ depending on a parameter $\alpha$ is said to be bounded if

$$
\left|C_{n_{1} \cdots n_{k}}^{j}(\alpha)\right| \leqq K_{n_{1} \cdots n_{k}}^{j}<\infty
$$

for all $\alpha$. Bochner and Martin prove that a coordinate system exists in which the elements of a bounded group take particularly simple form.

Their theorem is:

Any bounded group of inner transformations $T(\alpha)$ is similar to the group of its linear parts $L(T(\alpha))$ : there exists a transformation $S$ whose linear part is the identity

$$
S: x_{j}^{\prime}=x_{i}+\text { (higher powers), } \quad j=1, \cdots, k
$$

such that for all $\alpha$

$$
T(\alpha)=S^{-1} L(T(\alpha)) S .
$$

The question of the uniqueness of $S$ is not considered and this point is our concern here.

Now suppose that a bounded group of substitutions contains a

Received by the editors July 29, 1956 and, in revised form, November 16, 1956.

${ }^{1}$ S. Bochner and W. T. Martin, Several complex variables, Princeton, 1948, p. 19, Theorem 8. 
subgroup of linear transformations and let $A$ be any member of the subgroup.

Since $\cdots, A^{-n}, \cdots, A^{-1}, A^{0}, A, \cdots, A^{n}, \cdots$ is a bounded sequence of transformations

$$
A=H M H^{-1},
$$

where $M$ is the multiplication

$$
y_{j}=e^{i 2 \pi \alpha_{j}} x_{j}, \quad \alpha_{1}, \cdots, \alpha_{k} \text { real, }
$$

and $H$ is linear.

Now from the above theorem $A=S^{-1} A S$ i.e.

$$
S A=A S \text {. }
$$

Substituting from (1)

Writing

$$
H^{-1} S H M=M H^{-1} S H .
$$

$$
H^{-1} S H=V
$$

this becomes

$$
V M=M V
$$

where $V$ is of the form

$$
y_{j}=x_{j}+\sum_{n_{1}+\cdots+n_{k}>1} a_{n_{1} \cdots n_{k}}^{j} x_{1}^{n_{1}} \cdots x_{k}^{n_{h}} .
$$

Substituting from (2), $M V$ is the substitution

$$
y_{j}=e^{i 2 \pi \alpha_{j}} x_{j}+\sum_{n_{1}+\cdots n_{k}>1} a_{n_{1} \cdots n_{k}}^{j} e^{i 2 \pi \alpha_{j}} x_{1}^{n_{1}} \cdots x_{k}^{n_{k}}
$$

and $V M$ the substitution

$$
y_{j}=e^{i 2 \pi \alpha_{j}} x_{j}+\sum_{n_{1}+\cdots+n_{k}>1} a_{n_{1}}^{j} \cdots n_{k} e^{i 2 \pi\left(n_{1} \alpha_{1}+\cdots+n_{k} \alpha_{k}\right)} x_{1}^{n_{1}} \cdots x_{k}^{n_{k}} .
$$

Now (7) and (8) are identical if

i.e.

$$
a_{n_{1}}^{j} \cdots n_{k} e^{i 2 \pi \alpha_{j}}=a_{n_{1} \cdots n_{k}}^{j} e^{i 2 \pi\left(n_{1} \alpha_{1}+\cdots+n_{k} \alpha_{k}\right)}
$$

$$
a_{n_{1} \cdots n_{k}}^{j}=0 \text { if } n_{1} \alpha_{1}+\cdots+n_{k} \alpha_{k} \not \equiv \alpha_{j}(\bmod 1) .
$$

For most sets of $\alpha_{1}, \cdots, \alpha_{k}$, the equation in $n_{1}, \cdots, n_{k}$ 


$$
n_{1} \alpha_{1}+\cdots+n_{k} \alpha_{k}=\alpha_{j}(\bmod 1)
$$

will have no solution e.g. if $\alpha_{1}=e, \alpha_{2}=e^{2}, \cdots, \alpha_{k}=e^{k}$ (10) has no solution since $e$ is not algebraic. In such a case $V$ is the identity, consequently $S$ is also the identity and the subgroup of linear transformations is, in fact, the complete group. However for special sets of $\alpha_{1}, \cdots, \alpha_{k}, V$ need not be linear.

The above work gives information on the uniqueness of $S$ in the general case. If

$$
T=S_{1}^{-1} L(T) S_{1}
$$

and

$$
T=S_{2}^{-1} L(T) S_{2}
$$

then on writing $S_{2}=V S_{1}$ we find

$$
L(T)=V^{-1} L(T) V .
$$

If this last equation implies that the substitution $V$ (whose linear part is the identity) is the identity, then $S_{1}=S_{2}$ and $S$ is unique.

Here we are considering only a single transformation of the group and obviously to determine all restrictions on the arbitrariness of $S$ it may be necessary to consider the set of all elements of the group.

EXAMPLE. Let $\alpha_{1}=0, \alpha_{2}=1 / 2, H=I$.

Then (9) becomes

$$
\alpha_{n_{1} n_{2}}^{1}=0 \text { if } \frac{1}{2} n_{2} \not \equiv 0(\bmod 1), \quad \alpha_{n_{1} n_{2}}^{2}=0 \text { if } \frac{1}{2} n_{2} \not \equiv \frac{1}{2}(\bmod 1)
$$

$S=V$ can be any transformation of the form

$$
\begin{aligned}
y_{1} & =x_{1}+\sum_{n_{1}=0,1,2} \ldots \sum_{n_{2}=0,2,4} \ldots a_{n_{1}=0,1,2, \cdots} \sum_{n_{2}=1,3, \ldots}^{1} a_{n_{1} n_{2}} x_{1}^{n_{1}} x_{2}^{n_{2}}, x_{1}^{n_{1}} x_{2}^{n_{2}} .
\end{aligned}
$$

If the linear subgroup contains not only the substitution

$$
y_{1}=x_{1}, \quad y_{2}=-x_{2}
$$

but also the substitution

$$
y_{1}=-x_{1}, \quad y_{2}=x_{2}
$$

then $S$ must be the identity.

University of New England, Armidale, N.S.W., Australia 